\title{
Una metodología basada en el uso de indicadores para analizar la investigación realizada en una Institución Educativa
}

\section{A methodology based on the use of indicators to analyze the research carried out in an Educational Institution}

DOI: $10.46814 / 1 a j d v 4 n 1-019$

Recebimento dos originais: 03/01/2022

Aceitação para publicação: 15/02/2022

\author{
Alonso Perez-Soltero \\ Doctor en Tecnologías de la Información y las Comunicaciones \\ Institución: Universidad de Sonora \\ Dirección: Blvd. Luis Encinas y Rosales, Hermosillo Sonora, México \\ E-mail: aperez@industrial.uson.mx \\ Mario Barcelo-Valenzuela \\ Doctor en Tecnologías de la Información y las Comunicaciones \\ Institución: Universidad de Sonora \\ Dirección: Blvd. Luis Encinas y Rosales, Hermosillo Sonora, México \\ E-mail: mario.barcelo@unison.mx \\ Gerardo Sanchez-Schmitz \\ Doctor en Tecnologías de la Información y las Comunicaciones \\ Institución: Universidad de Sonora \\ Dirección: Blvd. Luis Encinas y Rosales, Hermosillo Sonora, México \\ E-mail: gerardo.sanchez@unison.mx

\section{Jose Luis Ochoa-Hernandez} \\ Doctor en Tecnologías de la Información y las Comunicaciones \\ Institución: Universidad de Sonora \\ Dirección: Blvd. Luis Encinas y Rosales, Hermosillo Sonora, México \\ E-mail: joseluis.ochoa@unison.mx

\section{David Alejandro Baca-Zepeda} \\ Ingeniero en Sistemas de Información \\ Institución: Universidad de Sonora \\ Dirección: Blvd. Luis Encinas y Rosales, Hermosillo Sonora, México \\ E-mail: davida.bacaz@correoa.uson.mx
}

\section{RESUMEN}

Las instituciones educativas, principalmente las de educación superior, requieren estar a la vanguardia; entre sus funciones principales se encuentran las actividades de docencia, vinculación e investigación. Para esta última, es importante que cuenten con información sobre la manera como la están desarrollando y el impacto que tiene en la sociedad. El objetivo del presente artículo es mostrar una metodología que contempla el uso de indicadores para llevar a cabo un análisis sobre la investigación realizada en el Departamento de Ingeniería Industrial de la Universidad de Sonora (UNISON), ubicada en México, durante el periodo 2000-2010. Como apoyo a la implementación de la metodología, se presenta una herramienta electrónica basada en Web en la cual los participantes en proyectos y 
publicaciones pueden registrarlos en la plataforma, además, cualquier interesado puede consultarla para conocer lo que se desarrolla en el Departamento en materia de investigación. Por otro lado, a través de esta herramienta informática, se pueden obtener reportes y gráficas representativas mediante el módulo de análisis de la información contenido en esta herramienta en base a los distintos parámetros que se contemplan. Estos análisis y resultados sirven para apoyar la toma de decisiones en instancias directivas del Departamento, así como detectar áreas de oportunidad en el rubro de investigación dentro del Departamento.

Palabras clave: indicadores, producción científica, metodología de análisis, prototipo informático.

\begin{abstract}
Educational institutions, mainly higher education, need to be at the knowledge frontier; its main functions include teaching, outreach and research activities. For the last one, it is important that they have information on how they are developing it and the impact it has on society. The aim of this paper is the development of a methodology that contemplates the use of indicators to carry out an analysis of the research carried out at the Department of Industrial Engineering at the Universidad de Sonora (UNISON), located in Mexico during the period of 2000-2010. As support for the implementation of the methodology, a Web-based electronic tool is presented in which the participants in projects and publications can document that information, in addition, any interested party can obtain it to know what is being developed in the Department in terms of research. On the other hand, through this computer tool, representative reports and graphs can be obtained through the analysis module of the information contained in this tool based on the different parameters that are considered. These analyzes and results serve for decision-making in the Department's management instances as well as detecting areas of research opportunities within the Department.
\end{abstract}

Keywords: indicators, scientific production, analysis methodology, informatic prototype.

\title{
1 INTRODUCCIÓN
}

En el contexto de este trabajo, es conveniente recordar que el proceso de la investigación científica contempla varias fases, las cuales, independientemente del tipo de investigación, se espera que al final del proceso se llegue a obtener un reporte de resultados [1]. De igual manera, esto es lo más relevante en este estudio, ya que interesa analizar y obtener un reporte de resultados de los productos de investigación generados dentro de un departamento de una institución de educación superior.

Por otro lado, no hay que olvidar que en el escenario actual altamente competitivo, se refuerza el discurso que la educación es un mecanismo de formación para el mundo del trabajo [2]. Asimismo, las instituciones de educación superior tienen la obligación de generar conocimiento y es una tarea de los investigadores poder llevarla a cabo. También se conoce que los resultados obtenidos de las investigaciones deben ser evaluables y medibles [3].

En la actualidad, existen diversas herramientas para el estudio de la producción científica, una de ellas es la bibliometría. Esta herramienta tiene como objetivo analizar y evaluar los resultados de la 
investigación, los cuales, impactan en los niveles de calidad de los programas educativos impartidos en una institución de educación superior [4].

En la institución en la que se lleva a cabo el estudio, los programas educativos se encuentran constantemente inmersos en procesos de evaluación, acreditación y re-acreditación, esto con el fin de mantener o incrementar su calidad. La acreditación es un proceso que llevan a cabo ciertos organismos acreditadores con el fin de ofrecer recomendaciones para mejorar la calidad de los servicios que ofrece un programa educativo [5]. Los programas tienen que acreditar un mínimo establecido de requerimientos que están organizados o incluidos en indicadores concretos de estos organismos, algunos de ellos fueron considerados para formar parte de este estudio.

El objetivo de este artículo es proponer una metodología basada en indicadores para recabar la información necesaria y con esta sea posible realizar un análisis de la investigación existente en el Departamento de Ingeniería Industrial, así mismo, sea posible validarla mediante el uso de herramientas electrónicas para la consulta y análisis de la información proveniente de los productos de investigación generados y registrados por los profesores e investigadores del Departamento.

Este trabajo está estructurado de la siguiente manera. Se inicia con los antecedentes y alcances del problema, a continuación, se describe el marco de referencia que sustenta el proyecto, después se describe la metodología propuesta basada en indicadores para realizar el análisis de la investigación. Posteriormente, se muestra la implementación de la metodología incluyendo los resultados obtenidos y finalmente, se describen las conclusiones.

\section{ANTECEDENTES Y ALCANCES DEL PROBLEMA}

El contexto de la problemática que se describe a continuación se ambienta en un escenario del año 2011, época en la cual la institución no contaba con herramientas tecnológicas y/o licencias de software para analizar su producción científica, además, la información no se encontraba registrada y concentrada en un solo lugar para facilitar su análisis. El Departamento de Ingeniería Industrial pertenece a la División de Ingeniería de la Universidad de Sonora (institución de educación superior ubicada en el noroeste de México), tiene a cargo tres programas de licenciatura que son: Ingeniería industrial y de Sistemas, Ingeniería en Sistema de Información e Ingeniería en Mecatrónica; y dos programas de posgrado que son: Ingeniería Industrial y la Especialización en Desarrollo Sustentable.

En cuanto a su planta académica, en ese momento estaba conformada por 46 profesores de tiempo completo y 56 profesores de tiempo parcial. Contaba con una población estudiantil de 1838 alumnos de licenciatura y 57 de posgrado. Este Departamento contaba con 8 academias las cuales eran: Manufactura y calidad, Tecnologías de información, Ingeniería sustentable, Diseño y control de 
sistemas, Gestión de capital humano, Modelación y optimización de sistemas, Ingeniería mecatrónica e Ingeniería estratégica.

Uno de los problemas que se tenían en el Departamento de Ingeniería Industrial, es que no se conocía de manera interna, ni se daba conocer de manera externa, los productos de investigación generados por parte de los académicos pertenecientes al departamento; se carecían de estadísticas del personal académico en cuanto a los proyectos de investigación y los productos derivados de ellos; además, tampoco se contaba con la información organizada que permitiera proporcionar estos resultados a diferentes organismo internos y/o externos a la institución.

Este estudio tuvo como alcance realizar un diagnóstico preliminar sobre la investigación realizada por el personal académico de tiempo completo del Departamento de Ingeniería Industrial, analizando, en una primera fase, únicamente los proyectos de la investigación concluidos de los cuales se ha entregado un informe final aprobado por el Consejo Divisional de Ingeniería; los concluidos que se encontraban registrados en organismos externos (y disponibles para su consulta en la Jefatura del Departamento) y las publicaciones en revistas y en congresos nacionales e internacionales disponibles en los informes oficiales de Rectoría y/o de la Dirección de Investigación y Posgrado de la Universidad de Sonora. Se incluye un diagnóstico de un periodo de 10 años, en este caso, del año 2000 al 2010. Una limitación de este estudio era que podían existir publicaciones de profesores-investigadores que no se encontraran registrados en los informes oficiales consultados.

\section{MARCO DE REFERENCIA}

A continuación, se definen algunos conceptos que son importantes para este trabajo. El término indicador está definido como un parámetro que se utiliza en el proceso evaluativo de cualquier actividad [6, 7]. Según la ONU, los indicadores son herramientas para clarificar y definir, de forma más precisa, objetivos e impactos. Son medidas verificables de cambio o resultado, diseñadas para contar con un estándar contra el cual evaluar, estimar o demostrar el progreso con respecto a metas establecidas, facilitan el reparto de insumos, produciendo productos y alcanzando objetivos [8]. Otro término que es preciso definir es el de bibliometría donde [6] menciona que es la colección, el manejo y análisis de manera cuantitativa de la información bibliográfica. Se usan estas herramientas con bases científicas para el estudio y evaluación de la producción científica, como es el caso de libros, artículos y revistas según especifican $[9,10]$.

Las herramientas tecnológicas son parte esencial hoy en día, más, si nos centramos en la web y de manera particular en la web 2.0, ya que en la web 1.0 únicamente éramos simples observadores [11, 12]. En la web 2.0 se permite la interacción y eso fue lo que le dio un atractivo revolucionario [13]. La creación de este tipo de web requiere conocer ciertos elementos básicos, como por ejemplo ¿Qué 
información requiere de entrada?, ¿Cuál será su funcionalidad?, ¿Cuál será su estructura?, ¿Cómo interactuaran los usuarios?, etc. y de manera ideal, saber ¿Cómo mantener actualizada la información? para que tenga un verdadero significado el uso de esta herramienta [14]. Existen diferentes lenguajes de programación para crear sitios Web, entre ellos se tienen HTML, JavaScript, ASP, Perl, Dreamweaver, PHP, entre otros.

PHP es uno de los lenguajes más extendidos en la Web. Se trata de un lenguaje de creación relativamente creciente que ha tenido una gran aceptación en la comunidad de Webmasters debido sobre todo a la potencia y simplicidad que lo caracterizó. PHP permite embeber los pequeños fragmentos de código dentro de la página HTML y realizar determinadas acciones de una forma fácil y eficaz sin tener que generar programas hechos íntegramente en un lenguaje distinto al HTML. PHP ofrece un sinfín de funciones para la explotación de bases de datos de una manera llana, sin complicaciones $[14,15]$.

Dreamweaver MX es un software fácil de usar que permite crear páginas web profesionales. Las funciones de edición visual de Dreamweaver MX permiten agregar rápidamente diseño y funcionalidad a las páginas, sin la necesidad de programar manualmente el código HTML. Como fue el caso de [16] para crear un proyecto virtual denominado Virtual Campus.

MySQL es la base de datos Open Source más usada a nivel mundial, se considera un sistema manejador de base de datos (o sus siglas en ingles DBMS) que trabaja con archivos llamados bases de datos y son del tipo relacional. Este manejador de base de datos se caracteriza por realizar los movimientos internos (altas, eliminaciones y modificaciones de datos y tablas) de manera rápida y con un buen rendimiento. También posee una baja probabilidad de corromper los datos, es decir, modificar los datos por un error del DBMS [17].

Las herramientas mencionadas anteriormente ayudan a crear sitios web los cuales según [18, 13] pretenden reemplazar a las aplicaciones de escritorio, es decir, eliminar el software instalado por servicios que estarán disponibles en la web implementados sobre servidores y serán accesibles simplemente a través de cualquier un navegador.

Por lo que el desarrollo de la herramienta tecnológica propuesta basada en la implementación de indicadores usando las nuevas tecnologías, augura el éxito y ayudará a estar a la vanguardia en el manejo de información bibliométrica en el departamento de estudio.

\section{METODOLOGÍA PROPUESTA}

Se propone una metodología basada en indicadores que se esquematiza en la figura 1. Esta forma de resolver el problema ofrece un valor agregado, ya que permite realizar análisis continuos y 
comparativos a través del tiempo, de acuerdo con indicadores acordes a las necesidades institucionales y del Departamento de Ingeniería Industrial.

Figura 1: Propuesta metodológica para el análisis de la investigación basada en indicadores.
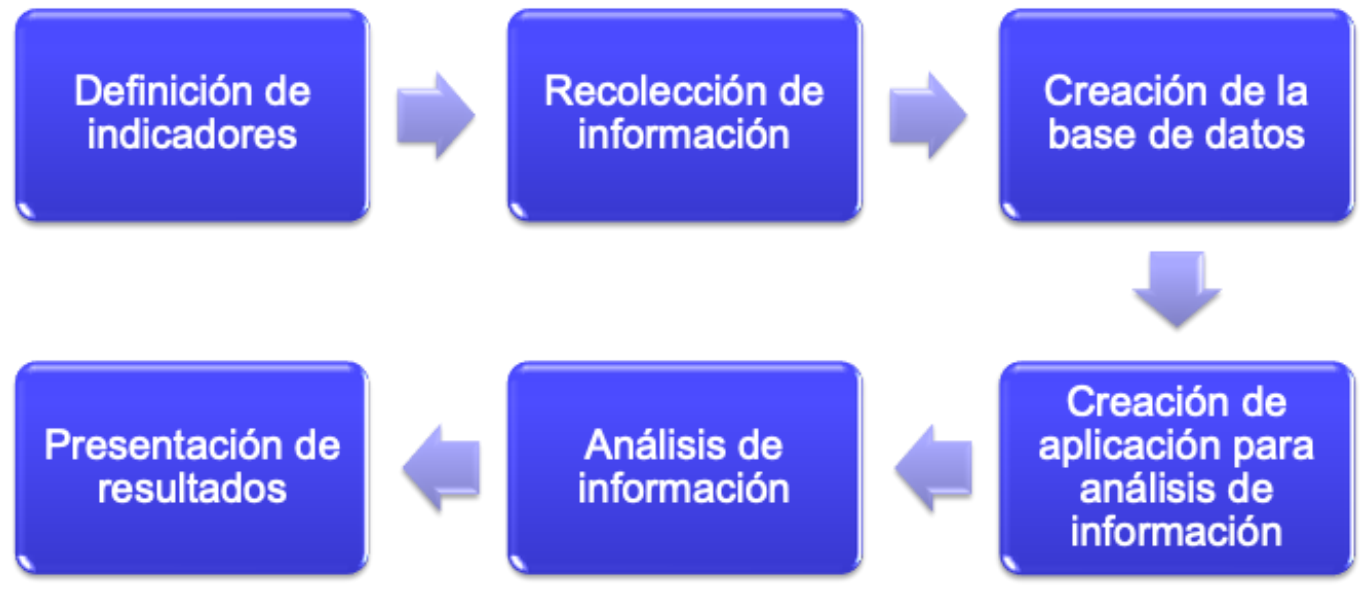

A continuación, se hace una muy breve descripción de cada uno de los pasos propuestos en la metodología:

Definición de indicadores: El objetivo de esta etapa es definir los indicadores que den la oportunidad de analizar de mejor manera la producción científica, así como su evolución en el tiempo y otros elementos dependiendo de la institución. Para definir indicadores es necesario tomar en cuenta los requisitos de organismos que evalúen la producción científica de la institución, leyes institucionales, organismos de evaluación al desempeño docente, así como las necesidades de las direcciones del área o departamento de la institución; las herramientas para poder definir estos indicadores son: una revisión bibliográfica, entrevistas o lluvia de ideas con las partes involucradas son importantes.

Recolección de información: En esta fase se determinan las posibles fuentes de donde se podía recolectar información para calcular los indicadores. La información se puede recabar en mayor parte gracias a las autoridades del área. La herramienta para obtener esta información es mediante una junta con los directores o los responsables de proporcionar esta información. Otras fuentes, son los portales o páginas de la institución y/o del departamento y la búsqueda en internet para información complementaria.

Creación de la base de datos: En esta fase se describe el diseño de la base de datos donde se guarda la información de los proyectos y publicaciones realizadas por parte del personal académico del Departamento de Ingeniería Industrial. Teniendo toda la información recolectada, se procede a diseñar la base de datos, la cual, permitió realizar consultas y obtener los indicadores definidos previamente. 
Creación de aplicación para análisis de información: En esta fase se realiza la implementación de la base de datos para que también pueda ser utilizada en ambiente Web. Esta fase contempla la creación de una interfaz mediante la cual los usuarios, puedan analizar mediante diferentes herramientas, la información almacenada en la base de datos

Análisis de información: Con ayuda de la aplicación de software, aquí se obtienen los valores de los indicadores, estos pueden ser por periodo de tiempo, por tipo de publicación (libro, capítulo de libro, artículo, ponencia, entre otros), por proyectos (con financiamiento, sin financiamiento, entre otros), por academias, por grupos de investigación, por tipo de participante (investigador, alumno, investigador externo, entre otros).

Presentación de resultados: Se muestran los diferentes tipos de indicadores para que la información pueda ser visualizada en forma de informes y/o mediante gráficas, y así apoyar la toma de decisiones en el departamento y proporcionar información requerida durante las evaluaciones llevadas a cabo por medio de los organismos evaluadores y/o acreditadores.

\section{IMPLEMENTACIÓN DE LA METODOLOGÍA}

Para validar la metodología propuesta, se diseñó e implementó un sistema de información basado en Web donde se almacena y muestra la información acerca de los proyectos de investigación concluidos de los profesores e investigadores, así como las publicaciones derivadas de ellos.

La información está disponible en un servidor Web, y está visible al público en general. La información se presenta de tal forma que también pueda ser consultada por los diversos interesados como: directivos del Departamento y de la Institución, personal académico, estudiantes, áreas internas y externas de la Institución, organismos evaluadores y de acreditación de programas educativos, entre otros.

A continuación, se detallan los pasos de la implementación de la metodología.

Definición de indicadores: Es una de las fases más importantes, ya que se recolectará información a partir de los indicadores que se definan, además de que estos indicadores influirán en la estructura de la base de datos, esto hace que otras fases dependan directamente de la definición de indicadores. Aquí se tomaron en cuenta los requisitos de organismos que evalúan la producción científica de la institución, reglamentos institucionales, organismos de evaluación al desempeño docente, organismos externos de acreditación de programas académicos, así como las necesidades de las direcciones del área o Departamento de la institución educativa. Lo anterior se hizo mediante revisiones de los documentos de estos organismos, en los cuales se definen los requerimientos de los programas en cuanto a diferentes rubros, entre ellos el de investigación. Los indicadores que representan necesidades específicas del Departamento se definieron a partir de juntas con los 
directivos, donde explicaron sus necesidades para contar con información que los apoyara, ya sea para la toma de decisiones o en la generación de informes para otras instancias de la institución. Los indicadores que se definieron fueron los siguientes: Número total de proyectos, número total de alumnos en proyectos, número de alumnos por proyectos, número total de publicaciones, proyectos y publicaciones según rangos de tiempo, programas educativos y academias.

Recolección de información: Para llevar a cabo este paso, se consultó información disponible en el portal institucional (informes anuales de rectoría), el portal de la División de Ingeniería (actas del consejo Divisional de Ingeniería), portal del Departamento de Ingeniería Industrial (página con el nombre de los profesores e investigadores) y directamente con directivos del Departamento de Ingeniería Industrial. En la primera fuente mencionada, los informes anuales del rector, se encontró información muy útil acerca de nombres de autores, nombres de responsables, departamento al que pertenecen estos, año de publicación, entro otros. El único problema que se presentó es que, había estadísticas totalizadas sobre proyectos y publicaciones (totales por tipo de proyecto, por tipo de investigación, por fuente de financiamiento) pero no se tiene acceso a información detallada acerca de todos los proyectos y publicaciones; por esta razón se acudió a la jefatura del Departamento, para que brindaran información detallada o información faltante en proyectos y publicaciones. En esta fase no es suficiente conseguir información respecto a los productos de la investigación, sino que también es necesario recolectar información adicional requerida por los indicadores. En este caso es necesario saber, la academia a la que pertenecen los profesores, los programas educativos donde participan, el tipo de rol que juegan los autores y los responsables, posición de autoría y rol en el proyecto (responsable o colaborador) respectivamente, el tipo de autor, si es alumno o profesor, entre otros.

Creación de la base de datos: Una vez definidos los indicadores y contando con varios ejemplos de productos de la información recolectada, se realizó el diseño de la base de datos. La estructura de la base de datos se hizo en MySQL, el manejador de base de datos del compendio de aplicaciones WampServer. Teniendo la estructura de las tablas y los campos bien definidos, se utilizó un software llamado "PHP Generator For MySQL", con el cual se creó una aplicación Web, para dar mantenimiento a la base de datos. En la aplicación se puede agregar, borrar, modificar y visualizar la información. Esta aplicación hace una conexión con el servidor donde se encuentra la base de datos, y la extrae para su gestión; cada tabla la hace una página Web donde se pueden definir restricciones en la manipulación de campos y registros.

Al tener una herramienta para crear la aplicación donde se gestiona la información de la base de datos, se crearon diferentes aplicaciones para los usuarios que interactúan con el sistema. Se definieron 3 tipos de usuarios basándose en el acceso a la información y los permisos que tendrá cada uno para el manejo de la misma. El usuario principal es el "administrador", el cual tiene acceso a toda 
la información y es el que está representado por la jefatura del departamento de Ingeniería Industrial. El administrador podrá agregar, editar y borrar información de cualquier tabla de la base de datos. Otro usuario es el de "autores", cada autor tiene su propia cuenta en el sistema, y el acceso de información está limitado para que sólo pueda ver y cambiar información concerniente a sus proyectos y publicaciones, así como a su información personal. Por último, el usuario con más restricciones en el sistema es el "público", el cual no tiene una cuenta, es para el público en general solo puede ver información general de los profesores, academias, programas educativos e información acerca de los autores, proyectos y publicaciones. Lo anterior es con la finalidad de divulgar información que puede ser de utilidad o interesar al personal académico, estudiantes, áreas internas y externas de la Institución, organismos evaluadores y de acreditación de programas educativos, empresas, organismos gubernamentales, o a cualquiera que se quiera informarse sobre lo que se hace en materia de investigación en el departamento de Ingeniería Industrial.

En la figura 2 se muestra una captura de pantalla de la aplicación Web donde se muestran las publicaciones de profesores del Departamento de Ingeniería Industrial.

Figura 2: Pantalla donde se muestran las publicaciones desde la sesión del administrador.

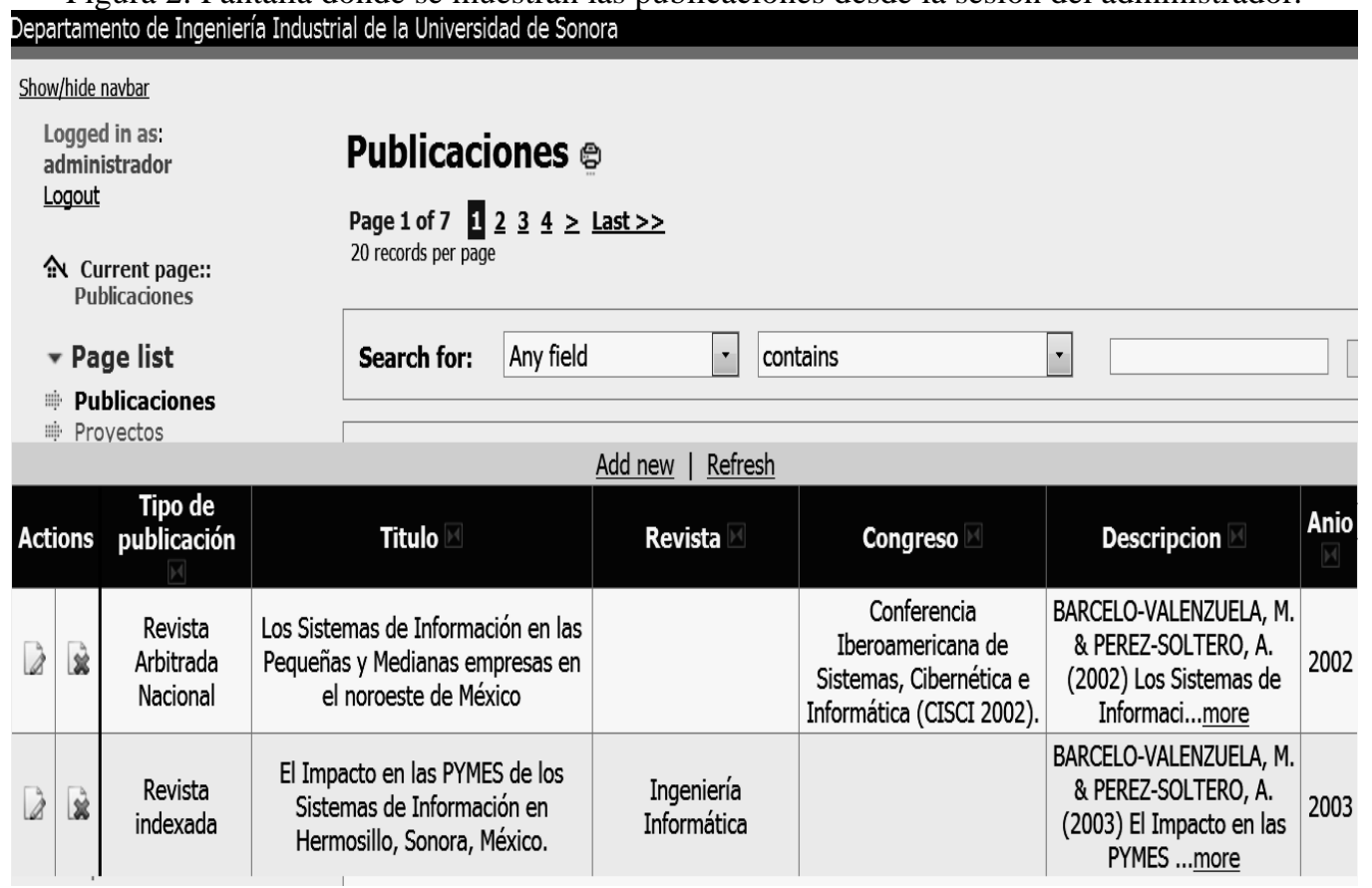

En la Figura 3 se muestra una vista de la sesión de un autor, y como se mencionó anteriormente, solo puede ver información concerniente a sus datos generales, publicaciones y proyectos. Como se observa, solo tiene acceso a 3 páginas, mientras que el administrador tiene acceso a 18 páginas, que son las 18 tablas definidas en la estructura de la base de datos. 
Figura 3: Pantalla para mostrar datos generales, proyectos y publicaciones desde la sesión de un autor.

\begin{tabular}{|c|c|c|c|c|c|c|c|c|c|c|c|c|}
\hline \multirow{2}{*}{\multicolumn{13}{|c|}{ 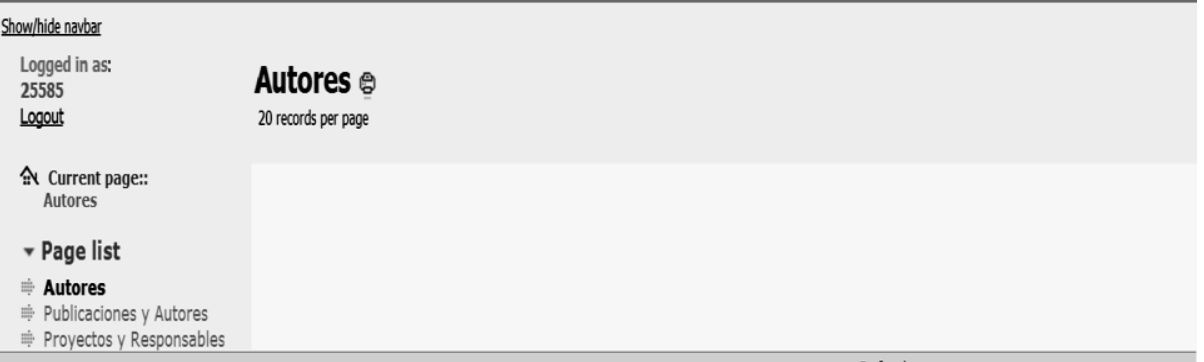 }} \\
\hline & & & & & & & & & & & & \\
\hline \multicolumn{13}{|c|}{ Refresh } \\
\hline Actions & Nombre & Cita & $\begin{array}{c}\text { Tipo } \\
\text { de } \\
\text { autor } \\
\text { D. }\end{array}$ & Categoria & $\begin{array}{c}\text { Licenciatura } \\
\square\end{array}$ & Maestria & Doctorado & Academia & $\begin{array}{c}\text { Programa } \\
1 \\
1\end{array}$ & $\begin{array}{l}\text { Programa } \\
2 \rrbracket\end{array}$ & Institucion & Email \\
\hline$\Leftrightarrow \mathbb{2}$ & \begin{tabular}{|l|} 
Perez \\
Soltero \\
Alonso
\end{tabular} & $\begin{array}{c}\text { Perez- } \\
\text { Soltero } \\
\text { A. }\end{array}$ & Profesor & MTC & $\begin{array}{l}\text { Ingeniero } \\
\text { Industrial y } \\
\text { de Sistemas }\end{array}$ & $\begin{array}{l}\text { Maestro en } \\
\text { Ciencias en } \\
\text { Tecrología } \\
\text { Informática }\end{array}$ & $\begin{array}{c}\text { Doctor en } \\
\text { Tecnologias de } \\
\text { la Información } \\
\text { y las } \\
\text { Comunicaciones }\end{array}$ & $\begin{array}{c}\text { Tecnologia } \\
\text { de } \\
\text { Informacion }\end{array}$ & ISI & MII & $\begin{array}{l}\text { Departamento } \\
\text { de Ingeniería } \\
\text { Industrial, } \\
\text { UNisoN }\end{array}$ & aperez@industrial.uson.mx \\
\hline
\end{tabular}

Ya que se tienen las aplicaciones para los diferentes tipos de usuarios, toda la información recolectada es almacenada en la base de datos.

Creación de aplicación para análisis de información: Para ello, se utilizaron diversas herramientas de software como Dreamwaver y la librería PHP “jpgraph”. Teniendo toda la información posible en la base de datos, se generó una aplicación para poder analizar esta información. Es una aplicación independiente a la creada para administrar la base de datos. En la figura 4 se muestra la primera pantalla de la interfaz de esta aplicación. Esta interfaz fue desarrollada en la aplicación Dreamweaver de Adobe Systems. Se recurrió a esta aplicación ya que la anterior "PHP Generator for MySQL" limitaba simplemente a hacer aplicaciones de base de datos donde solo puedes agregar, modificar, borrar registros, claro, también cuenta con seguridad y diseño en su presentación, pero no ofrecía la opción de generar una interfaz para crear las gráficas que se necesitan para el análisis de los indicadores propuestos.

Figura 4: Interfaz de aplicación para análisis de información.

\section{Sistema de indicadores}

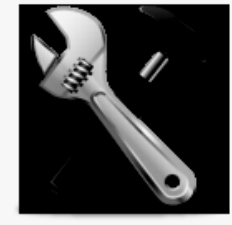

Mantenimiento

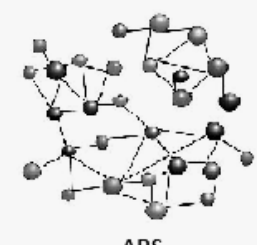

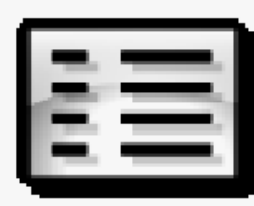

Reportes

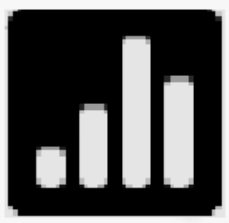

Graficas 
La interfaz cuenta con una liga al mantenimiento de la base de datos, se liga a las páginas para hacer el inicio de sesión de la otra aplicación. Cuenta también con una liga para acceder a los reportes que se generan para la jefatura del Departamento. La tercera liga que se muestra accede a una interfaz donde se cuenta con un sistema de filtros para generar gráficas, para el análisis de la información, este sistema de filtros se muestra en la figura 5. Además, se cuenta con una liga donde se está desarrollando otro proyecto sobre el análisis de las redes sociales de colaboración en materia de investigación.

Figura 5: Interfaz que muestran los filtros de generación de gráficas para el análisis de información.

\section{Gráficas}

\section{Por tipo \\ De: Publicación -}

\section{Por año}
De: Selecciona año
A: Selecciona año

\section{Por academia o programa}

academias

Diseño y Control de Sistemas

Gestión de Capital Humano

Ingeniería Estratégica

Ingeniería Mecatrónica

Ingeniería Sustentable

Manufactura y Calidad

Modelación y Optimización de Sistemas

Tecnologia de Informacion programas

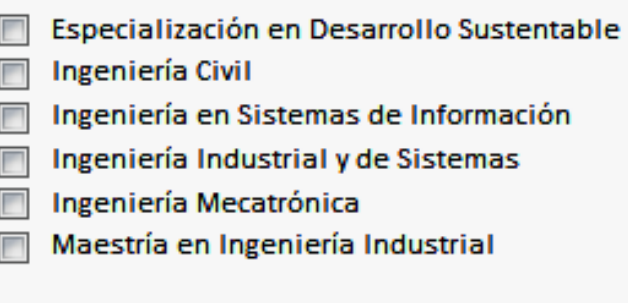

\section{Por tipo de autor}

De: Profesor

Para crear las gráficas se utilizó la librería PHP “jpgraph”, ya que es una herramienta fácil de entender y se adapta al entorno de la aplicación para el análisis de la información.

Análisis de información: Con estas y otras combinaciones, se obtiene información desglosada y detallada que permite hacer análisis y comparativos de la actividad de investigación dentro del Departamento de Ingeniería Industrial. Para el análisis de la información se utilizaron dos herramientas básicas, las gráficas y los reportes. Las gráficas se generan a partir de los filtros escogidos por el usuario. Los reportes son fijos, donde se muestra el comportamiento de los diferentes indicadores de interés, además, se cuenta con información adicional que la jefatura del Departamento debe entregar periódicamente a otras instancias en la institución. 
Presentación de resultados: Las gráficas se muestran dependiendo lo que el usuario escoja en el sistema de filtros, puede escoger rangos de tiempos, escoger por academias o programas educativos, si desea ver la producción dependiendo si es alumno o profesor, entre otros.

A continuación, se muestran algunos de los resultados obtenidos. El diagnóstico de la investigación realizada en el Departamento de Ingeniería Industrial, mostró resultados interesantes, entre ellos el que se observa en la figura 6, en ella se muestra el total de publicaciones que se han producido en el periodo 2000 - 2010 por los académicos del Departamento de Ingeniería Industrial.

Figura 6: Publicaciones realizadas por académicos del periodo 2000 - 2010.

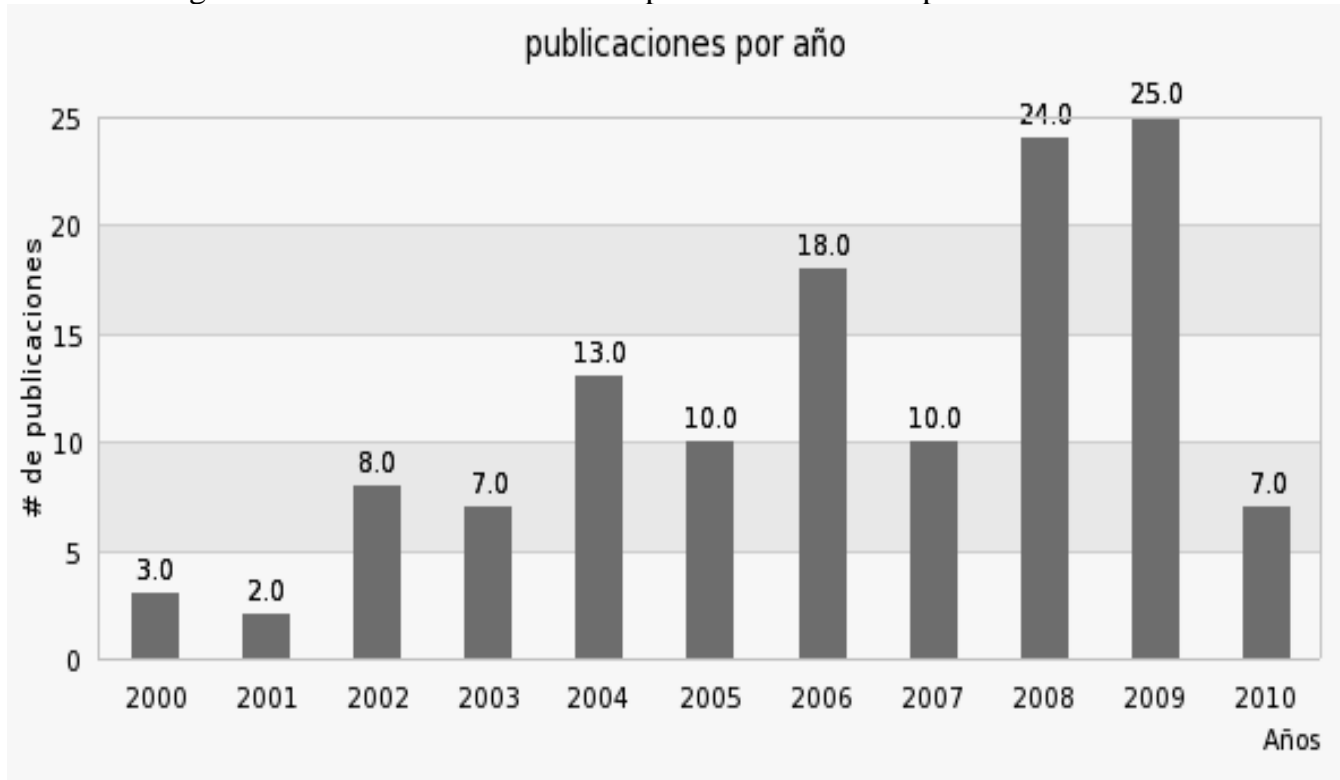

De acuerdo con la información analizada obtenida de los informes oficiales de la institución, durante el periodo 2000-2010 algunos de los principales resultados se describen a continuación: Las academias con mayor cantidad de publicaciones son las de Tecnologías de Información e Ingeniería Sustentable. El programa más beneficiado en cuanto a la planta de académicos que tienen producción científica es el de Ingeniería en Sistemas de Información. Los años con mayor número de publicaciones fueron los años 2008 y 2009. Del total de maestros de tiempo completo del Departamento, el 24\% tiene al menos una publicación. Del total de publicaciones, en el $2.3 \%$ de ellas, han participado estudiantes. En cuanto a proyectos llevados a cabo por académicos del Departamento, 2009 es el año que cuenta con el mayor registro, este rubro ha ido mejorando con los años. Las academias que cuentan con mayor cantidad de proyectos son Tecnologías de Información y Manufactura y Calidad. El programa con mayor cantidad de alumnos involucrados en proyectos es el programa de Ingeniería en Sistemas de Información. El programa de Ingeniería Industrial y de Sistemas es el que cuenta con mayor número de proyectos. Del total de proyectos, en el 10\% participaron alumnos adscritos a algún 
programa del Departamento. Se tiene registrados un total de 70 proyectos dentro del rango delimitado (2000-2010), cabe aclarar que solo se tienen proyectos registrados desde el año 2006. Publicaciones se tiene un total de 127 dentro del rango delimitado.

Como se mencionó anteriormente, una de las academias con más producción en el departamento, es la de Tecnologías de Información la cual cuenta con 59 publicaciones, lo que representa un $46 \%$ del total de las que se tienen almacenadas en la base de datos. En la figura 7 se muestra la producción de esta academia, en el rango de tiempo que se definió para este estudio.

Figura 7: Producción académica de la Academia de Tecnologías de Información del 2000-2010.

publicaciones por año

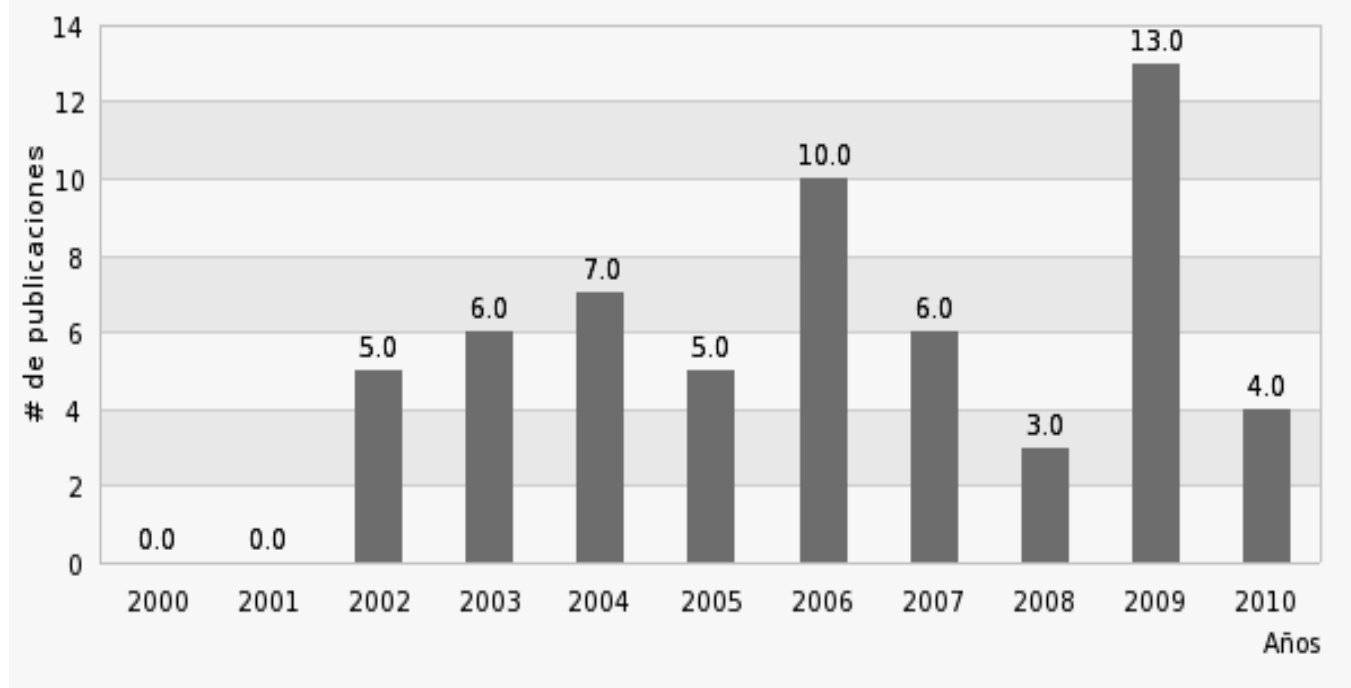

Como se puede observar, estos resultados dan una panorámica preliminar de cómo se encuentra la investigación en el Departamento de Ingeniería Industrial, si se desean los listados con los nombres de las publicaciones o de los proyectos, se cuenta con los reportes los cuales tienen información más detallada de los productos.

Es importante recalcar que la metodología y el sistema de información desarrollado, permiten analizar y dar seguimiento a los indicadores de manera confiable, sin embargo, por las limitaciones descritas en este estudio que contemplaba únicamente analizar la información disponible en los documentos oficiales consultados, los resultados reportados en este artículo pueden no reflejar en su totalidad lo que sucede en materia de investigación. Para resolver esta limitante, y gracias a que ahora se cuenta con la herramienta de software, basta con que todos los profesores-investigadores del departamento mantengan actualizada la información de sus publicaciones y/o proyectos concluidos. 


\section{CONCLUSIONES}

La herramienta tecnológica desarrollada para este trabajo fue un éxito, permitió descubrir información que no estaba a la mano y que no se conocía de manera directa. El uso de indicadores ayudó a visualizar y entender mejor el comportamiento interno del Departamento de Ingeniería Industrial con los resultados obtenidos, como ejemplo, el caso del conocer a la academia con mayor número de publicaciones, el programa educativo que tiene el mayor o menor número de publicaciones en base a la cantidad de académicos que tiene, el mejor año de publicación dentro del periodo establecido, el porcentaje de publicaciones que tiene cada investigador, en número de proyectos terminados, vigentes en los que participan alumnos, entre otros. Esta metodología realmente contribuyó al análisis de la investigación realizada en una institución de educación superior y a manera de conclusión se puede describir en los de siguientes aspectos: a) La propuesta de una metodología para el diagnóstico basada en indicadores para poder medir diferentes aspectos en las investigaciones realizadas por los cuerpos académicos, investigadores y profesores del Departamento de Ingeniería Industrial; b) El análisis de las áreas de investigación donde se tienen proyectos en curso, el número de profesores e investigadores que participan en cada una de las líneas de investigación y sus productos; c) La información disponible para apoyar a los procesos de acreditación de programas educativos realizados por organismos evaluadores externos y d) El mostrar a los diversos interesados, los resultados de las investigaciones hechas por los cuerpos académicos y profesores del departamento de ingeniería industrial para los fines que ellos requieran. 


\section{REFERENCIAS}

[1] Hernández Sampieri, R. y Mendoza Torres C. P., 2018. Metodología de la Investigación, 7ma ed. México: McGraw-Hill.

[2] Martins, D. M., 2021. Estado da arte das pesquisas sobre ENEM no Brasil: State of the art of research on ENEM in Brazil. Latin American Journal of Development, Vol. 3, No. 5, pp. 2898-2907. Disponible en: https://doi.org/10.46814/lajdv3n5-018

[3] Ramos, A., 2010. Ingeniería e innovación - Experiencia en investigación. Disponible en: https://www.iit.comillas.edu/documentacion/IIT-10-056A/Experiencia_en_investigación.pdf

[4] Macías-Chapula, C., 2001. Papel de la infometría y de la cienciometría y su perspectiva nacional e internacional. ACIMED Vol. 9, No. 4, pp. 35-41.

[5] Pérez, M., 1997. Evaluación, acreditación y calidad de la educación superior. Disponible en: http://publicaciones.anuies.mx/acervo/revsup/res098/txt2.htm

[6] Sancho, R., 1990. Indicadores bibliométricos utilizados en la evaluación de la ciencia y la tecnología. Revista Española de Documentación Científica, Vol. 13, No. 3-4, pp. 842-865.

[7] Llerena Paz, M. A., \& Arévalo Avecillas, M. E., 2021. Indicadores bibliométricos: origen, definición y aplicaciones científicas en el ecuador. Espíritu Emprendedor TES, Vol.5, No. 1, 130-153. Disponible en: https://doi.org/10.33970/eetes.v5.n1.2021.253

[8] ONU, 1999. Integrated and coordinated implementation and follow-up of major. United Nations conferences and summits. Nueva York, Estados Unidos de América, 10 y 11 de mayo de 1999

[9] Santos, R., \& Kobashi, N.Y., 2009. BIBLIOMETRIA, CIENTOMETRIA, INFOMETRIA: CONCEITOS E APLICAÇÕES.

[10] Moraes, L. \& Kafure, I., 2020. Bibliometria e ciência de dados. RDBCI: Revista Digital de Biblioteconomia e Ciência da Informação. Vol. 18. No. 00, pp. e020016. Disponible en: https://doi.org/10.20396/rdbci.v19i0.8658521.

[11] Lozano Salinas, J.M., 2008. La Web 2.0. Avances en supervisión educativa. Revista de la Asociación de Inspectores de Educación de España, ISSN 1885-0286, Nº 8.

[12] Firtan, M., 2008. Ajax - Web 2.0 para profesionales. México. Alfaomega.

[13] O'Reilly, Tim (2007): What Is Web 2.0: Design Patterns and Business Models for the Next Generation of Software. Published in: International Journal of Digital Economics No. 65: pp. 17-37.

[14] Hanze, H., Beatriz, X., Guerrero, V. \& Judith, S., 2010. Estudio de PHP y MySQL para el Desarrollo del Portal Web para el Municipio de Esmeraldas, Escuela superior politécnica de Chimborazo. Ecuador. Disponible en: http://dspace.espoch.edu.ec/handle/123456789/96

[15] Salazar, O.A., Aguirre, F.A., \& Osorio, J.A. (2011). Herramientas para el desarrollo rápido de aplicaciones web. Disponible en: https://doi.org/10.22517/23447214.511 
[16] Koyuncu, B., \& Kocabasoglu, P. 2008. Virtual campus. WSEAS International Conference. Proceedings. Mathematics and Computers in Science and Engineering. World Scientific and Engineering Academy and Society, Ankara, Turkey. Disponible en: http://www.wseas.us/elibrary/conferences/2008/hangzhou/musp/36-586-313.pdf

[17] Edward, L., 2006. MySQL, Second Edition. Peachpit Press.

[18] Pineda, J. G. H., 2009. Herramientas para la creación de un portal web 2.0. In Vestigium Ire, Vol. 2, No. 1. Disponible en: http://revistas.ustatunja.edu.co/index.php/ivestigium/article/view/93 